\title{
Hardness ratio evolutionary curves of gamma-ray bursts expected by the curvature effect
}

\author{
Y.-P. Qin ${ }^{1,2,3}$, C.-Y. $\mathrm{Su}^{2,4}$ J. H. Fan ${ }^{1}$, A. C. Gupta ${ }^{2,5 *}$
}

(Dated: August 17, 2017)

\begin{abstract}
We have investigated the gamma-ray bursts (GRBs) pulses with a fast rise and an exponential decay phase, assumed to arise from relativistically expending fireballs, and found that the curvature effect influences the evolutionary curve of the corresponding hardness ratio (hereafter HRC). We find, due to the curvature effect, the evolutionary curve of the pure hardness ratio (when the background count is not included) would peak at the very beginning of the curve, and then would undergo a drop-to-rise-to-decay phase. In the case of the raw hardness ratio (when the background count is included), the curvature effect would give rise to several types of evolutionary curve, depending on the hardness of a burst. For a soft burst, an upside-down pulse of its raw HRC would be observed; for a hard burst, its raw HRC shows a pulse-like profile with a sinkage in its decaying phase; for a very hard burst, the raw HRC possesses a pulse-like profile without a sinkage in its decaying phase. For a pulse-like raw HRC as shown in the case of the hard and very hard bursts, its peak would appear in advance of that of the corresponding light curve, which was observed previously in some GRBs. For illustration, we have studied here the HRC of GRB 920216, GRB 920830 and GRB 990816 in detail. The features of the raw HRC expected in the hard burst are observed in these bursts. A fit to the three bursts shows that the curvature effect alone could indeed account for the predicted characteristics of HRCs. In addition, we find that the observed hardness ratio tends to be harder at the beginning of the pulses than what the curvature effect could predict and be softer at the late time of the pulses. We believe this is an evidence showing the existence of intrinsic hard-to-soft radiation which might be due to the acceleration-to-deceleration mode of shocks.
\end{abstract}

PACS numbers: $98.70 . \mathrm{Rz}, 98.80 .-\mathrm{k}$

\footnotetext{
${ }^{* 1}$ Center for Astrophysics, Guangzhou University, Guangzhou 510006, P. R. China

${ }^{2}$ National Astronomical Observatories/Yunnan Observatory, Chinese Academy of Sciences, Kunming
} 
650011, P. R. China

${ }^{3}$ Physics Department, Guangxi University, Nanning 530004, P. R. China

${ }^{4}$ Department of Physics, Guangdong Industry University, Guangzhou 530004, P. R. China

${ }^{5}$ Tata Institute of Fundamental Research, Homi Bhabha Road, Colaba, Mumbai 400 005, India; Electronic address: ypqin@ynao.ac.cn, alok@ynao.ac.cn 


\section{INTRODUCTION}

Because of high output rate of observed radiation, gamma-ray bursts (GRBs) are assumed to undergo a stage of fireballs which expand relativistically $[1,2]$. Relativistic bulk motion of the gamma-ray-emitting plasma would lead to some phenomena of GRBs [3]. To account for the observed spectra of the bursts, the Doppler effect over the whole fireball surface would play an important role [4-7].

There is a consensus that the prompt emission of GRBs might arise from nonthermal synchrotron radiation [8,9], inverse Compton emission [10], and others such as thermal, saturated Comptonization [11]. Among these mechanisms, the nonthermal synchrotron is likely to be the dominant one but this mechanism alone could not account for the spectra observed in many GRBs. It is expected that in any case several mechanisms are at work, the spectra would become complicated. Generally, a more flexible one, the so-called Band function [12] which is purely an empirical spectral form, is adopted to describe the observed spectra of most of the bursts.

Some simple bursts with well-separated structure suggest that they may consist of fundamental units of emission such as pulses, with some of them being seen to possess a FRED form [13]. These FRED pulses could be well represented by some empirical or semi-empirical functions, and with these functions many statistical properties of GRB pulses were revealed $[14,15]$. Phenomena of FRED pulses were interpreted as signatures of the curvature effect [6,15-18]. The effect arises from the emission of the expanding surface of fireballs. Photons emitted from different parts of the surface could reach a distant observer at different times. The delay of time is determined not only by the difference of the corresponding emission time but also by the variation of the distance of the emitting areas to the observer when their angles to the line-of-sight are different. Meanwhile, in the case that the angles to the line-of-sight of the emitting areas are different, the Doppler shifting of the photons will differ

significantly. Combining all possible effects which are due to the expending fireball surface comes to the full content of the curvature effect (see a detailed analysis in [6]).

Light curves of GRBs in different energy channels differ significantly in both the width and height of the curves [13]. In the past few years, various attempts of interpretation of the light curves have been made [14-17,19]. When fitting different energy channel light curves, the different free parameters were allowed due to the difference in appearances of 
the curves. Based on these fits the temporal scale factors of a given pulse measured at different energies were found to be related to the corresponding energies by a power law [14,20-21]. It was suspected that this behavior might result from a relative projected speed or a relative beaming angle [21]. Due to the similar observed features, the difference between different channel light curves of GRBs might probably be due to the observed energy channel itself. In other words, light curves of different energy channels might arise from the same mechanism, with the only difference being the energy range concerned. Indeed, as shown in [18], four channel light curves of GRB 951019 could be well fitted by the same mechanism (the same local pulse and the same rest frame spectrum) when the curvature effect is taken into account. Other recent investigations of the properties of FRED pulses of GRBs such as, the width of GRB pulses as a power law function of energy, the characteristics of the profile of FRED pulses in the decaying phase, the spectral lag as a consequence of the curvature effect, and the relationship between the power law index and the local pulse width of FRED pulses were explored by [22-27]. Recently, some authors attempted to explain the X-ray tails and flares observed soon after the prompt emission of some GRBs with the curvature effect [28].

The study of the spectral evolution of GRBs could be traced back to as early as 1980s, where softening of the spectra with time as a general phenomenon, the so-called hard-to-soft phenomenon was found [29]. Recently, quantitative studies of the spectral behavior of GRB pulses were made by many authors [17,30-33]. It was found that the hardness-intensity correlation obtained from the statistical analysis was regarded as a result of the spectral evolution, could be accounted for by the curvature effect [17]. However, as a direct evidence of the spectral evolution, the evolutionary curve of the spectral peak energy or the hardness ratio of GRB pulses should be intensely explored and it would be necessary to check if the curvature effect influences these curves.

In this paper, we study the evolution of the hardness ratio due to the fact that the data of this quantity are available at time bins as small as $64 \mathrm{~ms}$ in the BATSE catalogue. In this way, the details of the evolutionary curve would be well illustrated. We first explore how the curvature effect influences such theoretical curves for some typical bursts, and then choose three FRED pulse sources to study this issue in order to check if individual hardness ratio evolutionary curves could be accounted for by the curvature effect. 


\section{THEORETICAL PREDICTION}

As suggested by many authors, the profile of GRB FRED pulses could be well accounted for by the curvature effect [15-18]. We wonder what characteristics of the HRC of FRED pulses could be expected when the curvature effect is at work.

The formula employed is equation (21) presented in [18] which could be written as

$$
C(\tau)=C_{0} \frac{\int_{\widetilde{\tau}_{\theta, \min }}^{\widetilde{\tau}_{\theta, \max }}\left[\widetilde{I}\left(\tau_{\theta}\right)\left(1+\beta \tau_{\theta}\right)^{2}\left(1-\tau+\tau_{\theta}\right) \int_{\nu_{1}}^{\nu_{2}} \frac{g_{0, \nu}\left(\nu_{0, \theta}\right)}{\nu} d \nu\right] d \tau_{\theta}}{\left(1+\frac{\beta}{1-\beta} \tau\right)^{2}}
$$

with $\tau_{\min } \leq \tau \leq \tau_{\max }, \tau_{\min } \equiv(1-\beta) \tau_{\theta, \min }, \tau_{\max } \equiv 1+\tau_{\theta, \max }, \tau \equiv\left(t-D / c+R_{c} / c-t_{c}\right) /\left(R_{c} / c\right)$, and $\tau_{\theta} \equiv\left(t_{\theta}-t_{c}\right) /\left(R_{c} / c\right)$, where $\theta$ is the angle to the line of sight, $t$ is the observation time measured by the distant observer, $t_{\theta}$ is the local time measured by the local observer located at the place encountering the expanding fireball surface at the position denoted by angle $\theta$ and the radius of the fireball $R=R\left(t_{\theta}\right), t_{c}$ is the initial local time, $R_{c}$ is the radius of the fireball measured at $t_{\theta}=t_{c}, D$ is the distance from the fireball to the observer, $\widetilde{I}\left(\tau_{\theta}\right)$ represents the development of the intensity measured by the local observer, and $g_{0, \nu}\left(\nu_{0, \theta}\right)$ describes the rest frame radiation, and $\nu_{0, \theta}=(1-\beta+\beta \tau) \Gamma \nu /\left(1+\beta \tau_{\theta}\right)$, $\widetilde{\tau}_{\theta, \min }=\max \left\{\tau_{\theta, \min },\left(\tau-1+\cos \theta_{\max }\right) /\left(1-\beta \cos \theta_{\max }\right)\right\}$ and $\widetilde{\tau}_{\theta, \max }=\min \left\{\tau_{\theta, \max },(\tau-1+\right.$ $\left.\left.\cos \theta_{\min }\right) /\left(1-\beta \cos \theta_{\min }\right)\right\}$, with $\tau_{\theta, \min }$ and $\tau_{\theta, \max }$ being the upper and lower limits of $\tau_{\theta}$, which confine $\widetilde{I}\left(\tau_{\theta}\right)$, and $\theta_{\max }$ and $\theta_{\min }$ being the upper and lower limits of $\theta$, which confine the emission area, respectively. With this formula, count rates of both spherical fireballs (when $\theta_{\min }=0$ and $\theta_{\max }=\pi / 2$ ) and uniform jets (when $\theta_{\min }=0$ and $\theta_{\max }<\pi / 2$ ) could be calculated as long as the local emission intensity and the rest frame spectrum are assumed.

The spectral hardness ratio was previously defined as the ratio of the count rate of a higher energy channel to that of a lower energy channel $[29,34]$. We adopt this definition since it could be evaluated within a time interval of $64 \mathrm{~ms}$ and thus one can compare the theoretical curves with the observational data of BATSE sources. The observed count subtracting the background count within a $64 \mathrm{~ms}$ interval for BATSE sources might become zero or negative due to fluctuation. In this case, the hardness ratio would be ill-defined. To avoid this difficulty, we simply study a raw hardness ratio which is defined as the observed count of a higher energy band divided by that of a lower band, where the background counts are not subtracted (in contrast to this, the ratio of the observed count subtracting the background count of a higher energy band to that of a lower band is called a pure hardness ratio). 
Following previous studies, we define the raw hardness ratio as $H R_{3 / 2} \equiv C_{3} / C_{2}$, where $C_{3}$ and $C_{2}$ are the expected or observed counts of the third and second BATSE channels, respectively. The corresponding bi-channel light curves which is defined as $C_{2+3} \equiv C_{2}+C_{3}$ will also be studied so that a direct comparison between the hardness ratio curve and the light curve could be made.

An expected value of count rates when taking into account the background would be calculated with the light curve of (1) plus a background count rate function (the expected background curve), and the expected value of the raw hardness ratio would be calculated with this kind of count rate.

To reveal the main characteristics of the HRCs expected from the curvature effect, we temporarily ignore possible effects arising from any of the following factors: the real forms of the local pulse and the rest frame spectrum, the development pattern of the rest frame spectrum, and the variation mode of the Lorentz factor. We assume an exponential rise and exponential decay local pulse

$$
\widetilde{I}\left(\tau_{\theta}\right)=I_{0}\left\{\begin{array}{rr}
\exp \left(\frac{\tau_{\theta}-\tau_{\theta, 0}}{\sigma_{r}}\right) & \left(\tau_{\theta, \min } \leq \tau_{\theta} \leq \tau_{\theta, 0}\right) \\
\exp \left(-\frac{\tau_{\theta}-\tau_{\theta, 0}}{\sigma_{d}}\right) & \left(\tau_{\theta, 0}<\tau_{\theta}\right)
\end{array}\right.
$$

emitting with a rest frame Comptonized radiation form

$$
g_{0, \nu}\left(\nu_{0, \theta}\right)=\nu_{0, \theta}^{1+\alpha_{0, C}} \exp \left(-\nu_{0, \theta} / \nu_{0, C}\right) .
$$

We assign $\tau_{\theta, 0}=10 \sigma_{r}+\tau_{\theta, \min }$ so that the interval between $\tau_{\theta, 0}$ and $\tau_{\theta, \min }$ would be large enough to make the rising part of the local pulse close to that of the exponential pulse. Note that, $\tau_{\theta, \min }$ could be freely chosen so long as it satisfies the following constraint: $\tau_{\theta, \min }>$ $-1 / \beta[18]$. Without any loss of generality we take $\tau_{\theta, \min }=0$.

The expected observed light curves in BATSE channels 2 and 3 are assumed to be

$$
C_{2, o b}(\tau)=C_{2}(\tau)+\eta_{2} \frac{C_{2+3, p}}{2}
$$

and

$$
C_{3, o b}(\tau)=C_{3}(\tau)+\eta_{3} \frac{C_{2+3, p}}{2}
$$

where $C_{2}(\tau)$ and $C_{3}(\tau)$ are determined by $(1)$ [note that $C_{2}(\tau)$ and $C_{3}(\tau)$ are different in the limits of the integral of energy in equation (1)], $C_{2+3, p}$ is the peak of $C_{2+3}(\tau)$, and $\eta_{2}$ and $\eta_{3}$ are constants which denote the magnitude of the background count of channels 2 

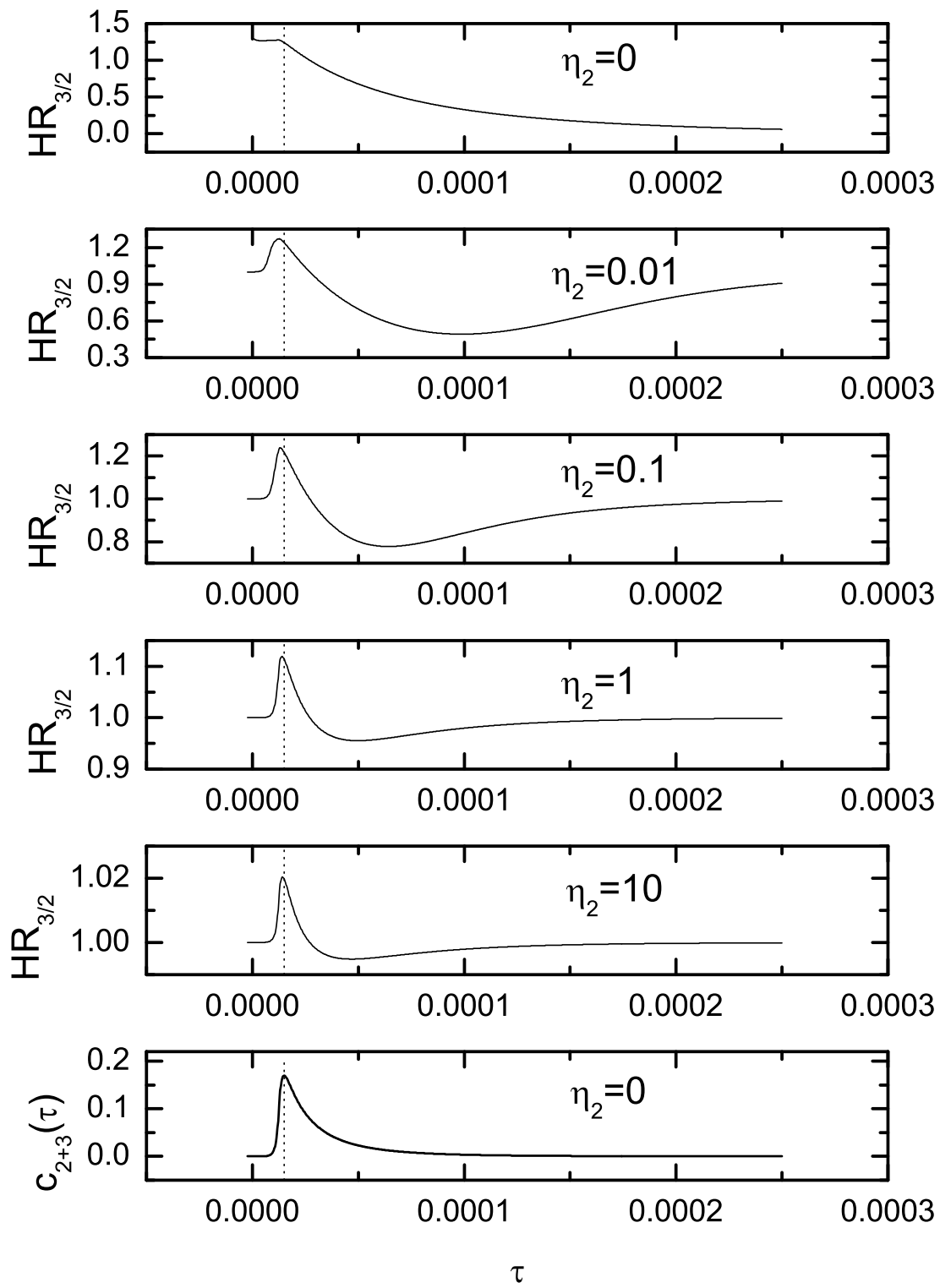

FIG. 1: Plot of the hardness ratio $H R_{3 / 2}$ vs. the relative observation time $\tau$ for various values of $\eta_{2}$ (from top, the first to the fifth panels) in the case of adopting $\Gamma=200$ and $\eta_{3}=\eta_{2}$, where equations (1)-(5) are employed and we take $\sigma_{r}=0.1, \sigma_{d}=\sigma_{r}, \alpha_{0, C}=-0.6, \nu_{0, C}=0.55 \mathrm{keVh}^{-1}$, and $C_{0}=1$. Plotted in the last panel is the corresponding bi-channel light curve, where background counts are not included. The dotted lines in all panels mark the time when the peak count of the light curve presented in the last panel is observed. 

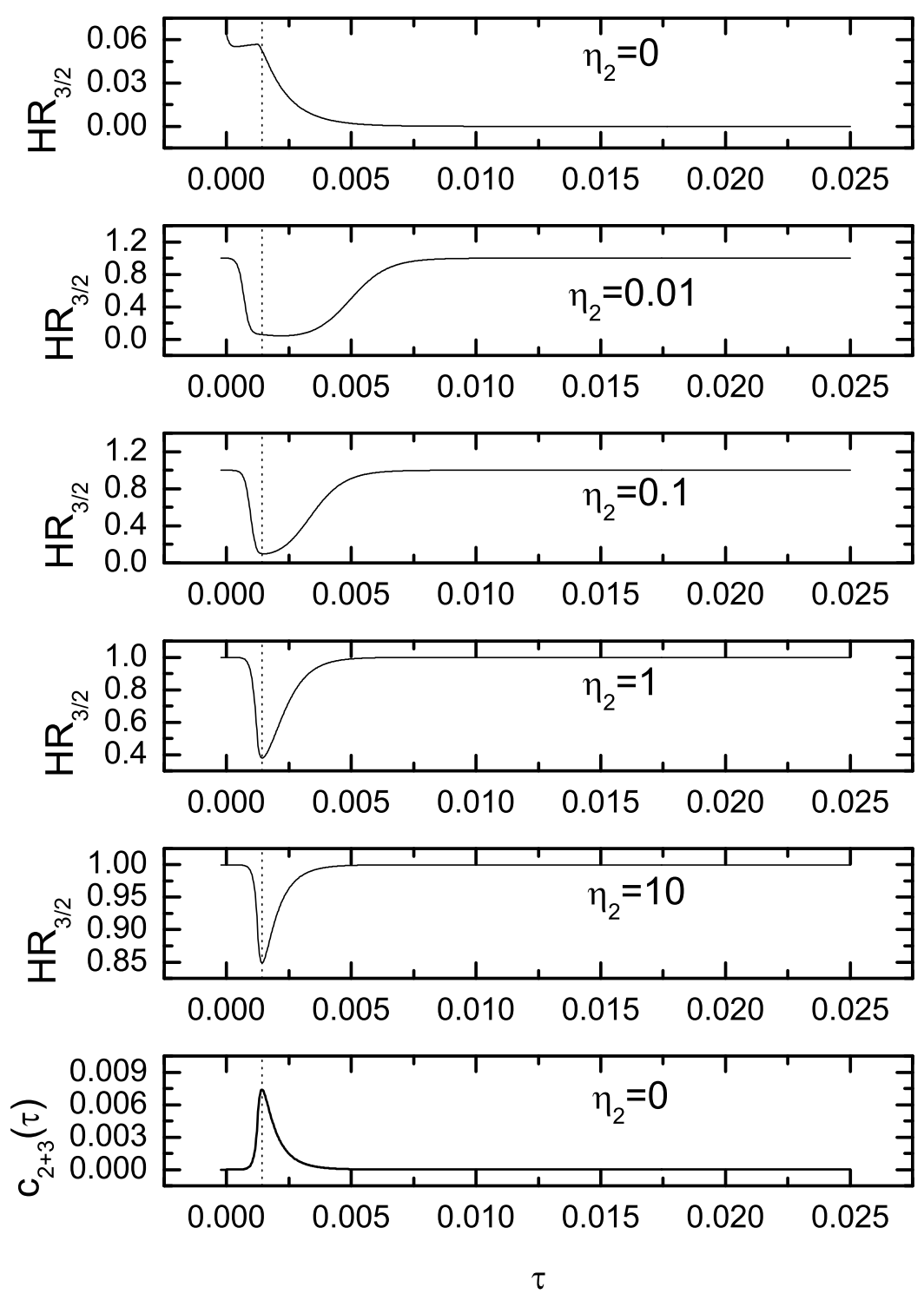

FIG. 2: Plot of the curves of Fig. 1 when calculating them by adopting $\Gamma=20$ instead of $\Gamma=200$.

and 3 relative to $C_{2+3, p}$ respectively. Here, for the sake of simplicity, we assume that the background count does not evolve with time. Following [23], we adopt $\alpha_{0, C}=-0.6$ and $\nu_{0, C}=0.55 \mathrm{keVh}^{-1}$ and consider a typical hard and typical soft bursts with $\Gamma=200$ and $\Gamma=20$ respectively. In addition, a typical very hard burst with $\Gamma=2000$ is also considered.

Displayed in Fig. 1 are the HRCs for the typical hard burst $(\Gamma=200)$ in the case of 

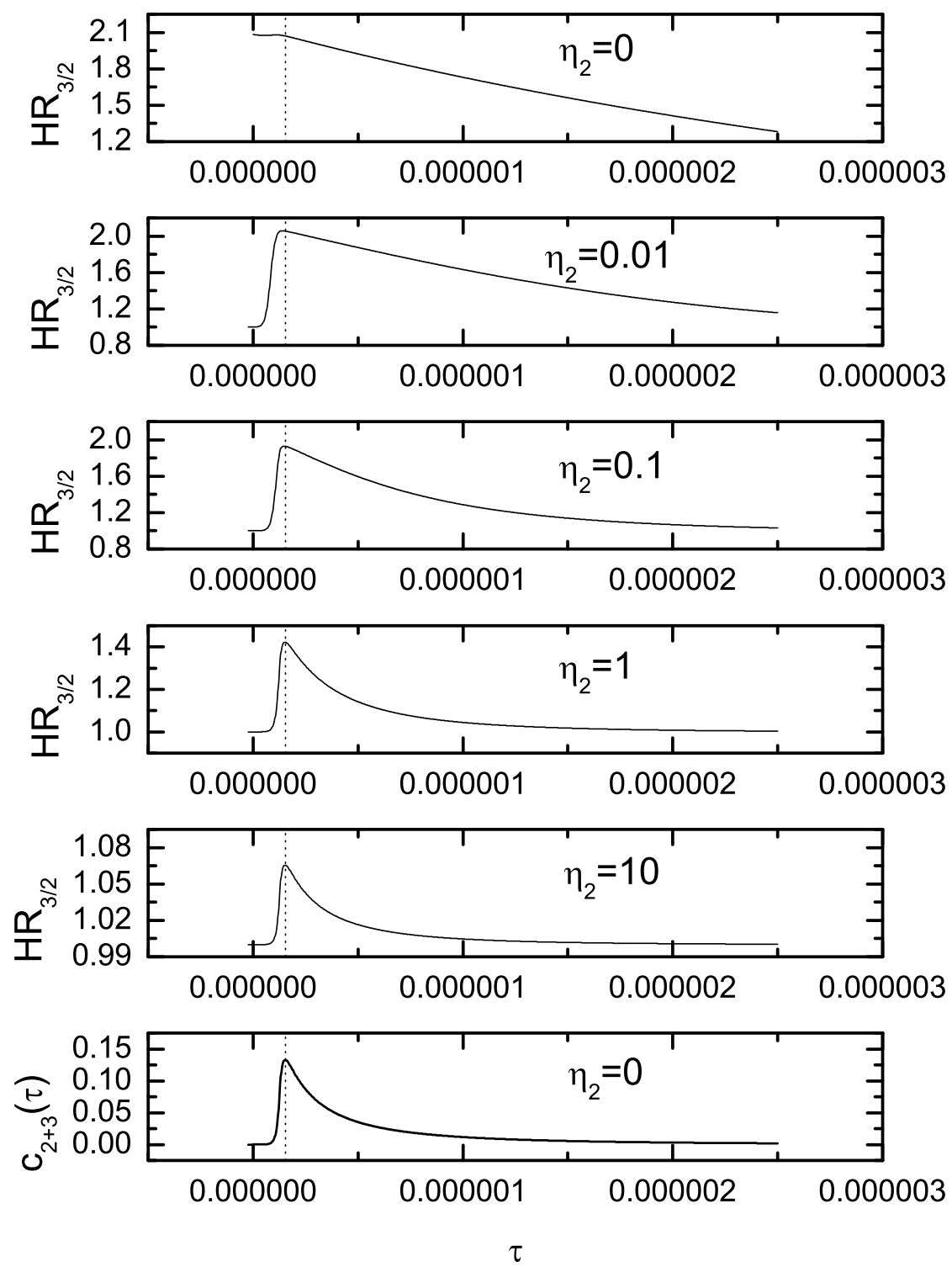

FIG. 3: Plot of the curves of Fig. 1 when calculating them by adopting $\Gamma=2000$ instead of $\Gamma=200$.

$\eta_{3}=\eta_{2}$ for various values of $\eta_{2}$. When taking $\Gamma=20$ and $\Gamma=2000$ we get Figs. 2 and 3 for the typical soft and typical very hard bursts respectively in the same case. For the pure hardness ratio, the peaks of HRCs of the three typical bursts are 0.063 (for the typical soft burst), 1.3 (for the typical hard burst) and 2.1 (for the typical very hard burst). We find from 
these figures that: a) for the signal alone (i.e., when the background count is not included), the hardness ratio (here, the pure hardness ratio) would peak at the very beginning of the light curve, and then would undergo a drop-to-rise-to-decay phase, for the three bursts; b) for the typical soft burst, an absorption-line-feature pulse (called an upside-down pulse) is observed in its raw HRCs (i.e., when the background count is included), and the larger the background count, the narrower the upside-down pulse; c) for the typical hard burst, a pulse-like profile with a sinkage in its decaying phase would be observed in its raw HRCs, and the larger the background count, the shallower the sinkage; d) for the typical very hard burst, a pulse-like profile without a sinkage in its decaying phase would be observed in its raw HRCs, and the larger the background count, the narrower the pulse; e) in the case of the two hard bursts, the peak of the raw HRCs would appear in advance of that of the corresponding bi-channel light curve; f) for the three typical bursts, the profile of the raw HRCs would depend strongly on the ratio of the background count to the signal count.

To find out how background counts in the two channels are at work, we calculate the HRCs of the typical hard burst in cases of $\eta_{3}=0.8 \eta_{2}$ and $\eta_{3}=1.2 \eta_{2}$ as well. The results are presented in Fig. 4. We find that the profile as well as the magnitude of the raw HRCs would also depend on the ratio between the background counts in the two corresponding channels.

In Table 1, we have listed the peak time and width of the HRCs and light curves of the typical hard burst in the case of adopting $\sigma_{d}=\sigma_{r}$ and various sets of parameters. In calculating the width of the HRCs, we consider only the portion of the curves where the values of the hardness ratio are larger than that arising merely from the background counts (e.g., the flat section shown in the beginning of the HRCs in Figs. 1 and 4). For the pure HRCs, since they are not a pulse-like curve, the width is not available.

Adopting $\sigma_{d}=5 \sigma_{r}$, we find the same characteristics observed above, suggesting that the characteristics do not come from the form of local pulses.

To investigate how the rest frame spectrum affects HRCs, we repeat the above analysis by replacing the adopted Comptonized radiation form with a Band function spectrum [12]

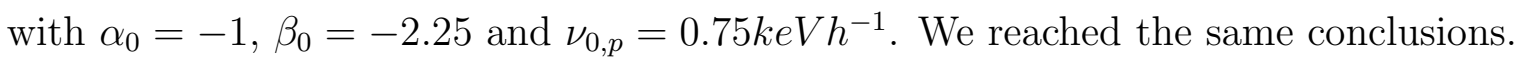


TABLE I: Peak time and width of the HRCs and light curves of the typical hard burst in the case of adopting $\sigma_{d}=\sigma_{r}$ and various sets of parameters

\begin{tabular}{|c|c|c|c|c|c|}
\hline curve & $\sigma_{r}$ & $\eta_{3}$ & $\eta_{2}$ & $\tau_{\text {peak }}$ & $F W H M_{\tau}$ \\
\hline$C_{2+3}$ & 0.1 & 0 & 0 & $1.49 \times 10^{-5}$ & $1.36 \times 10^{-5}$ \\
\hline$H R_{3 / 2}$ & 0.1 & 0 & 0 & $2.50 \times 10^{-8}$ & - \\
\hline \multirow[t]{4}{*}{$H R_{3 / 2}$} & 0.1 & $0.8 \eta_{2}$ & 0.01 & $1.27 \times 10^{-5}$ & $1.72 \times 10^{-5}$ \\
\hline & & & 0.1 & $1.34 \times 10^{-5}$ & $1.41 \times 10^{-5}$ \\
\hline & & & 1 & $1.42 \times 10^{-5}$ & $1.09 \times 10^{-5}$ \\
\hline & & & 10 & $1.44 \times 10^{-5}$ & $9.60 \times 10^{-6}$ \\
\hline \multirow[t]{4}{*}{$H R_{3 / 2}$} & 0.1 & $\eta_{2}$ & 0.01 & $1.27 \times 10^{-5}$ & $1.21 \times 10^{-5}$ \\
\hline & & & 0.1 & $1.32 \times 10^{-5}$ & $9.60 \times 10^{-6}$ \\
\hline & & & 1 & $1.39 \times 10^{-5}$ & $8.08 \times 10^{-6}$ \\
\hline & & & 10 & $1.42 \times 10^{-5}$ & $7.32 \times 10^{-6}$ \\
\hline \multirow[t]{4}{*}{$H R_{3 / 2}$} & 0.1 & $1.2 \eta_{2}$ & 0.01 & $1.24 \times 10^{-5}$ & $7.07 \times 10^{-6}$ \\
\hline & & & 0.1 & $1.27 \times 10^{-5}$ & $4.80 \times 10^{-6}$ \\
\hline & & & 1 & $1.32 \times 10^{-5}$ & $3.79 \times 10^{-6}$ \\
\hline & & & 10 & $1.34 \times 10^{-5}$ & $3.79 \times 10^{-6}$ \\
\hline$C_{2+3}$ & 1 & 0 & 0 & $1.44 \times 10^{-4}$ & $9.34 \times 10^{-5}$ \\
\hline$H R_{3 / 2}$ & 1 & 0 & 0 & $2.50 \times 10^{-7}$ & - \\
\hline \multirow[t]{4}{*}{$H R_{3 / 2}$} & 1 & $0.8 \eta_{2}$ & 0.01 & $1.27 \times 10^{-4}$ & $1.21 \times 10^{-4}$ \\
\hline & & & 0.1 & $1.32 \times 10^{-4}$ & $9.85 \times 10^{-5}$ \\
\hline & & & 1 & $1.39 \times 10^{-4}$ & $7.32 \times 10^{-5}$ \\
\hline & & & 10 & $1.42 \times 10^{-4}$ & $6.57 \times 10^{-5}$ \\
\hline \multirow[t]{4}{*}{$H R_{3 / 2}$} & 1 & $\eta_{2}$ & 0.01 & $1.27 \times 10^{-4}$ & $9.09 \times 10^{-5}$ \\
\hline & & & 0.1 & $1.29 \times 10^{-4}$ & $6.82 \times 10^{-5}$ \\
\hline & & & 1 & $1.34 \times 10^{-4}$ & $5.56 \times 10^{-5}$ \\
\hline & & & 10 & $1.37 \times 10^{-4}$ & $5.05 \times 10^{-5}$ \\
\hline \multirow[t]{4}{*}{$H R_{3 / 2}$} & 1 & $1.2 \eta_{2}$ & 0.01 & $1.24 \times 10^{-4}$ & $5.30 \times 10^{-5}$ \\
\hline & & & 0.1 & $1.27 \times 10^{-4}$ & $3.54 \times 10^{-5}$ \\
\hline & & & 1 & $1.29 \times 10^{-4}$ & $2.53 \times 10^{-5}$ \\
\hline & & & 10 & $1.32 \times 10^{-4}$ & $2.27 \times 10^{-5}$ \\
\hline
\end{tabular}




\section{OBSERVATIONAL CHARACTERISTICS OF THE HARDNESS RATIO EVO- LUTIONARY CURVE SHOWN IN THREE FRED PULSE BURSTS}

Since FRED pulses of GRBs are likely to suffer the curvature effect [6,15-18,22-25], we suspect that the characteristics predicted above can be checked by the observational data of the HRCs of such GRBs. Here, we study the development of the hardness ratio for three bursts whose light curves are that of FRED and then check if the features predicted above are observed. The three bursts are chosen to investigate the issue due to the following reasons: a) if a characteristic is common for most FRED pulses it would likely be observed in fewer cases; b) if the curvature effect plays a role in the hardness ratio evolutionary curve of a few bursts it would probably play a role in other bursts of the same kind; c) as a primary investigation, we concern in this paper only the characteristics of the hardness ratio evolutionary curve revealed by individual pulses rather than concern a statistical property of the bursts.

The bursts studied are GRB 920216 (\#1406), GRB 920830 (\#1883), and GRB 990816 (\#7711). Count rates of the bursts are available in the website of BATSE $[35,36]$, where the counts within the $64 \mathrm{~ms}$ bin for four energy channels $[25,55] \mathrm{keV},[55,110] \mathrm{keV},[110,320] \mathrm{kev}$, and $[>320] \mathrm{keV}$ are presented.

Presented in the BATSE website, one also finds the values of $T_{90}$, the duration of a source, for the three bursts. We extend the signal data of the sources from $T_{90}$ to $2 T_{90}$, starting from $T_{90} / 2$ previous to the start time of the duration. Let this range be denoted by $t_{\min } \leq t \leq t_{\max }$. Data beyond this range (that of $t<t_{\min }$ and $t>t_{\max }$ ), which are independent of the signal data, are employed to find the fit to the count rate of the background.

The duration as well as the range of the $2 T_{90}$ signal data of the three bursts are as follows.

GRB 920216: $T_{90}=19.968 s, t_{\min }=-9.28 s, t_{\max }=30.656 s$.

GRB 920830: $T_{90}=17.344 s, t_{\min }=-8.288 s, t_{\max }=26.4 s$.

GRB 990816: $T_{90}=20.928 s, t_{\min }=-10.016 s, t_{\max }=31.84 s$.

The developments of the raw hardness ratios $H R_{3 / 2}$ and the bi-channel light curves $C_{2+3}$ of the three bursts are shown in Fig 5. Several characteristics could be observed from the figure, with some of them being reported previously [14,29,34,37]: a) the raw hardness ratio as a function of time exhibits a pulse shape; b) the peak of the raw hardness ratio $H R_{3 / 2}$ appears in advance of the peak of the bi-channel light curve $C_{2+3}$; c) the width of the HRC 
is narrower than that of the corresponding light curve; d) there is a sinkage in the decay phase of the HRC. All these features are in agreement with what predicted in the case of the typical hard burst (see Fig. 1). Note that, the third character could be observed in the 4th and 5th panels counting from the top in Fig. 1. It corresponds to the case of relatively large background counts.

\section{CURVATURE EFFECT AS A KEY FACTOR ACCOUNTING FOR THE HARDNESS RATIO EVOLUTIONARY CURVE}

As long as the characteristics observed in the three FRED pulse bursts are in agreement with what predicted by the curvature effect, we try to make a fit to the HRCs of these bursts based on the assumption that the curvature effect is important. The formula employed is equation (1).

One could observe from Fig. 5 that the hardness ratio data are quite randomly scattered. This is due to the much scatter of the count rate data. There are several ways to ease this scattering. One is to smooth the count rate data before the hardness ratio is calculated. Another is to deal with the data associated with larger bins of time. With the former method one could maintain the large number of data points but the information of signal would be somewhat influenced, while with the latter method the information of signal would not be affected, but the number of data points would be reduced. As there are enough data points for the three bursts, we prefer the latter approach.

The background data (that of $t<t_{\min }$ and $t>t_{\max }$ ) would be fitted with a quadratic polynomial, and then this fitting curve would be applied to the signal interval and would be taken as the expected background count rate there (instead of finding it from websites, we perform the fit to the background count ourselves since data of larger bins are dealt with in this paper). An expected value of the count rate within the signal interval when taking into account the background would be calculated with the light curve of (1) plus the background count rate function (the background fitting curve), and the expected value of the raw hardness ratio would be calculated with this kind of count rate.

To check if the curvature effect could play an important role in producing the HRC characteristics observed in the three bursts, we temporarily ignore possible effects arising from the real forms of the local pulse, the rest frame spectrum, the development pattern 
of the rest frame spectrum, or the variation mode of the Lorentz factor. We therefore assume and employ the local pulse (2) and rest frame spectrum (3) to perform the fit. Due to the same reasons, some parameters adopted above are maintained, i.e., we assign $\tau_{\theta, 0}=10 \sigma_{r}+\tau_{\theta, \min }$ and take $\tau_{\theta, \min }=0$ (note that, the origin of time would be determined by $t_{0}$ presented below, and thus $\tau_{\theta \text {,min }}$ could be freely chosen).

As shown in [38], the opening angle of jets could be as small as $\theta=0.03$. When applying equation (1), we take $\theta_{\max }=0.03$ so that one could find if the HRCs observed above could arise from uniform jets with such a small opening angle when the curvature effect is taken into account. Since the profile of light curves is not significantly affected by the Lorentz factor [18], we simply take $\Gamma=200$. To relate the observation time $t$ and the relative time scale $\tau$, we assign $t=t_{1} \tau+t_{0}$. Since the background counts are included in the definition of the raw hardness ratio, the magnitude of counts, $C_{0}$, could not be cancelled. Therefore, there would be seven free parameters determined by fit, which are the rest frame peak energy $E_{0, C}$ and spectral index $\alpha_{0, C}$, the two widths of the exponential rise and exponential decay local pulse $\sigma_{r}$ and $\sigma_{d}$, the two time constants $t_{1}$ and $t_{0}$, and the count magnitude $C_{0}$. Note that the fitted parameters such as the Lorentz factor are merely parameters of the model. They are not the real parameters of the bursts concerned. In particular, adopting $\Gamma=200$ as a parameter of the model does not imply that the matter concerned must have such a large speed of motion.

We know that both the HRC and bi-channel light curve in Fig. 5 are determined by the light curves of the second and third channels. If the curvature effect is at work, it should affect not only the second and third channel light curves but also the first and fourth channel light curves. We thus regard relations arising from the four channel light curves calculated with equation (1) as those associated with the curvature effect (here, as stated above, other possible effects are ignored). Therefore, the observed four channel light curves of the bursts will be fitted simultaneously with equation (1) to find out if and how the observed HRC could be accounted for by the curvature effect.

As shown in Fig. 5, the observed light curve data are quite randomly scattered. We assume that fluctuation could be responsible to this scattering. Based on this assumption, the statistics $\chi^{2}$ associated with a light curve could be defined as

$$
\chi^{2} \equiv \sum_{i=1}^{n} \frac{\left(C_{o b, i}-C_{i}\right)^{2}}{C_{i}}
$$


where $n$ is the total number of bins, $C_{o b, i}$ and $C_{i}$ are the observed and expected values of the count respectively, within the $i$-th bin. The $\chi^{2}$ of the light curve could then be calculated with equation (6). As four channels are involved, we define a combined $\chi^{2}$ to perform the fit. Let the combined $\chi^{2}$ be $\chi_{c o m}^{2}=\sum_{j=1}^{4} \chi_{j}^{2}$, where $\chi_{j}^{2}$ is the $\chi^{2}$ associated with the fit to the $j$-th channel light curve. The numbers of bins for the four light curves are the same for each source, and therefore the total number of data for $\chi_{c o m}^{2}$ is $4 n$ (note that data of different channels are independent). The best fit would be obtained when the smallest value of $\chi_{\text {com }}^{2}$ is reached. In this way, the four channel light curves could be "simultaneously" fitted.

\section{GRB 920216:}

There are 7504 data points in total for GRB 920216. We divide this range of data uniformly into 1876 bins with each bin containing four $64 \mathrm{~ms}$ time intervals. The quadratic polynomial fits to the background counts yield: $0.256 C(t)=752-0.0812 t-0.000500 t^{2}$ $(P<0.0001)$ (channel 1); 0.256C $(t)=588+0.0394 t-0.000462 t^{2}(P<0.0001)$ (channel $2) ; 0.256 C(t)=394-0.0602 t-0.000201 t^{2}(P<0.0001)($ channel 3$) ; 0.256 C(t)=205+$ $0.0122 t+0.000106 t^{2}(P<0.0001)$ (channel 4). The fit to the four channel light curves produces: $E_{0, C}=0.191 \mathrm{keV}, \alpha_{0, C}=0.398, \sigma_{r}=0.298, \sigma_{d}=0.181, t_{1}=2.98 \times 10^{5} \mathrm{~s}$, $t_{0}=-10.1 s$, and $C_{0}=1.90 \times 10^{4}$ photon. The reduced $\chi^{2}$ of the fit is: $\chi_{\text {com, } \nu}^{2}=2.85$.

\section{GRB 920830:}

There are 7504 data points in total for GRB 920830 as well. In the same way we divide this range of data uniformly into 1876 bins. The quadratic polynomial fits to the background counts yield: $0.256 C(t)=879-0.0493 t-0.000242 t^{2}(P<0.0001)($ channel 1$) ; 0.256 C(t)=$ $604-0.0233 t-0.000118 t^{2}(P<0.0001)($ channel 2$) ; 0.256 C(t)=425-0.0407 t+0.000108 t^{2}$ $(P<0.0001)$ (channel 3); 0.256C $(t)=234-0.0568 t+0.000175 t^{2}(P<0.0001)$ (channel

4). The fit to the four channel light curves produces: $E_{0, C}=0.220 \mathrm{keV}, \alpha_{0, C}=0.467$, $\sigma_{r}=0.489, \sigma_{d}=0.469, t_{1}=5.10 \times 10^{4} s, t_{0}=-2.76 s$, and $C_{0}=1.44 \times 10^{4}$ photon. The reduced $\chi^{2}$ of the fit is: $\chi_{\text {com, } \nu}^{2}=3.10$.

\section{GRB 990816:}

The number of data points for GRB 990816 is 5856. We divide this range of data uniformly into 1464 bins. The quadratic polynomial fits to the background counts yield: $0.256 C(t)=961-0.0529 t-0.000160 t^{2}(P<0.0001)($ channel 1$) ; 0.256 C(t)=689+$ $0.0362 t+0.0000328 t^{2}(P<0.0001)($ channel 2$) ; 0.256 C(t)=626+0.265 t+0.000211 t^{2}$ $(P<0.0001)\left(\right.$ channel 3); $0.256 C(t)=426+0.354 t+0.000150 t^{2}(P<0.0001)$ (channel 
4). The fit to the four channel light curves produces: $E_{0, C}=0.166 \mathrm{keV}, \alpha_{0, C}=0.824$, $\sigma_{r}=0.0916, \sigma_{d}=0.111, t_{1}=2.95 \times 10^{5} s, t_{0}=-3.03 s$, and $C_{0}=3.13 \times 10^{5}$ photon. The reduced $\chi^{2}$ of the fit is: $\chi_{\text {com }, \nu}^{2}=2.74$.

As shown in Fig. 6, we present both the observed HRCs and the expected HRCs which are deduced from the four fitting light curves, together with two corresponding multi-channel light curves of the three sources.

One finds that the curvature effect could indeed produce the characteristics of HRCs observed in the three bursts. Besides this, we find that the observed hardness ratio tends to be harder at the beginning of the pulses than what the curvature effect could predict (which is represented by the corresponding fitting curves) and softer at the late time of the pulses. This is called a "harder-leading" problem. It indicates that if we believe that the curvature effect is important as assumed above, then besides the curvature effect there might be other effects being at work. Nevertheless, as the figure shows, although it is possible that there exist some other effects, the curvature effect could indeed be a key factor accounting for the characteristics the observed HRCs of the three bursts show.

Pure hardness ratio curves of the three sources deduced from the four fitting light curves of each burst are shown in Fig. 7. One finds that, in the three cases, when the "harderleading" problem is ignored the pure hardness ratio curve would be hardest at the very beginning and then would undergo a short period of drop-to-rise and later would decay continuously until the pulse dies away, as predicted above. The short period of drop-to-rise is a characteristic of the curvature effect. It is unclear if this characteristic still holds when the mechanism leading to the "harder-leading" problem is at work. However, we believe that this characteristic could be expected in cases when the "harder-leading" problem is very insignificant. We wonder if one could check this with some other sources where the scattering of data is small enough so that the pure hardness ratio could be well measured at the very beginning of the pulses.

As pointed out by [17], the phenomenon of the softening of the spectra with time revealed by statistical analysis is quite general which is independent of any changes in the intrinsic spectrum and therefore independent of the physical environment where the pulses are produced. The observed phenomenon is generally in agreement with the evolutionary curve of the pure hardness ratio illustrated in Fig. 7. But in terms of the raw hardness ratio, we find a soft-to-hard-to-soft feature instead. This is a characteristic associated with the 
drop-to-rise-to-drop feature shown in the evolutionary curve of the pure hardness ratio (see Fig. 7), and therefore the two features are alternatives which could serve as an indicator of the curvature effect (note that they are not independent).

\section{DISCUSSION AND CONCLUSIONS}

The first question put forward is that: are the three bursts isolated cases showing the characteristics predicted by the curvature effect? This could be easily answered when one examines the HRC for more bursts. Listed in the tables of [26] are seven other FRED pulse bursts, GRB910721 (\#563), GRB920925 (\#1956), GRB930214c (\#2193), GRB930612 (\#2387), GRB941026 (\#3257), GRB951019 (\#3875) and GRB951102B (\#3892), which were also intensely studied elsewhere [18,21]. Let us show the HRC curve for these bursts and examine their characteristics.

Fig. 8 shows the HRC as well as the corresponding bi-channel light curve of the seven bursts. We find that, for GRB910721, GRB930214c, GRB930612, GRB941026, the characteristics predicted by the typical hard burst are observed (see Fig. 1, the 4th and 5th panels counting from the top). For GRB920925 and GRB951019, a possible sinkage feature is observed, although the fluctuation of background is very large. Compared with the theoretical predictions, these two bursts might be relatively soft (see Fig. 2). This conjecture could be checked if one is able to remove or largely ease the great fluctuation observed in their HRCs (by the smooth of data or other approaches; the issue is beyond the scope of this paper). For GRB951102B, no signals are detected due to the great chaos and therefore we cannot tell if the curvature effect is or is not at work. We suspect that this might not only be due to fluctuation, but also be due to the small signal it might possibly possess in its HRC.

The second question concerns that what causes the so-called "harder-leading" problem. It should be reminded that several other possible effects are ignored in the above analysis. Among them there is an economic one accounting for this effect, which is the development of the rest frame spectrum when it is harder, itself, at the beginning and softer at the late time of the pulses. The mechanism leading to this seems expectable. In the case of a Comptonized radiation, electrons of the inner shell that scattering photons in the outer shell would possess a larger value of speed relative to the expanding fireball surface at the early time of shocks and a smaller value later. In the case of a synchrotron radiation, electrons 
that radiate are expected to gain larger acceleration at the beginning of a shock than at later times. Obviously, how the rest frame spectrum evolves depends on what mechanisms dominate in the corresponding period.

In this paper, we use a Comptonized spectrum instead of the Band function to perform the fit due to several reasons. The first is that the Comptonized spectrum could indeed represent some GRB spectra [39]. The second is that we would like to know if a real mechanism could be employed to describe (even roughly) the observed HRC. The third is that there are only two free parameters in the Comptonized spectrum, while in the Band function form there are three. From Fig. 6 one finds that, since the fit is quite satisfied, ignoring the possible effect arising from the deviation of the Comptonized spectrum from the real rest frame one may not give rise to a severe problem. And it seems unlikely that this effect could lead to the "harder-leading" problem so long as there is a more economic mechanism accounting for the latter.

It is noticed that, the peak energy alone cannot determine the hardness ratio (to determine the hardness ratio, one needs to know the peak energy as well as the low and high energy indexes). Any of the two quantities could not replace the other. The development of the hardness ratio reflects only one aspect of the spectral evolution, while that of the spectral peak energy would reflect another aspect. Thus, to understand the development of the spectrum in a more detail, it is necessary to study the evolutionary curve of the peak energy as well. We expect that such an investigation could independently test the curvature effect.

There has been a consensus that due to the relativistic aberration of light, isotropically emitted radiation in the co-moving frame will be beamed into a cone with opening angle $\theta \sim 1 / \Gamma$ and therefore only photons emitted from the fireball surface within this cone will be detectable by the observer [40]. However, as shown in [18], emission from a cone of opening angle $\theta=1 / \Gamma$ would lead to a FRED pulse light curve with a cutoff tail (when the local pulse is extremely narrow) or a turnover feature (when the local pulse is not so narrow). It suggests that contribution of the emission from the area of $\theta>1 / \Gamma$ to the tail of the light curve is important (while it is indeed negligible in the main part of the light curve; see Figs. 1 and 2 in [18]). This effect might have an impact on the HRC. In this paper, we take $\theta=0.03$ according to [38], which is much larger than $1 / \Gamma=0.005$ for $\Gamma=200$ adopted in this paper. Since the effects associated with $\theta=0.03$ and $\theta=\pi / 2$ are not distinguishable, the conclusions obtained in this paper hold for both cases of a spherical fireball and an 
uniform jet when its opening angle is large enough.

The plan is that when a more flexible form of local pulses is adopted, one will be able to obtain a better fit. However, the evidence of curvature effect presented above is obvious enough. The fact that adopting local pulse (2) can simply account for the observed data of the three bursts when merely taking into account the curvature effect shows explicitly that the effect could indeed serve as the main cause of the observed HRC characteristics of some bursts, especially for those with FRED pulses.

It is obvious that an intrinsic hard-to-soft spectral evolution could account for the phenomenon of the spectral softening with time which were generally observed in GRBs. Thus, the above analysis suggests only that the curvature effect is a plausible origin of the phenomenon. To tell if a burst suffers effects other than the curvature one, one needs to investigate if any of these effects could give rise to the characteristics the light curve and the HRC of the burst possesses. This will take some time and it deserves.

Based on the above analysis and discussion, the main conclusions of this paper are as follows. Due to the curvature effect, the evolutionary curve of the pure hardness ratio would peak at the very beginning of the curve, and then would undergo a drop-to-rise-to-decay phase. In the case of the raw hardness ratio, the curvature effect would give rise to several types of evolutionary curve, depending on how hard is a burst. For the typical soft burst with $\Gamma=20$ (when assuming a rest frame Comptonized radiation with indexes of $\alpha_{0, C}=-0.6$ and $\nu_{0, C}=0.55 \mathrm{keVh}^{-1}$ ), an upside-down pulse would be observed; for the typical hard burst with $\Gamma=200$, a pulse-like profile with a sinkage in its decaying phase would be observed; for the typical very hard burst with $\Gamma=2000$, a pulse-like profile without a sinkage in its decaying phase would be observed. In the case that the raw hardness ratio evolutionary curve is a pulse-like one, as shown in the typical hard and very hard bursts, its peak would appear in advance of that of the corresponding light curve. The features of raw HRCs predicted in the case of the typical hard burst are observed in GRB 920216, GRB 920830, and GRB 990816. A fit to these bursts shows that the curvature effect alone could indeed account for the characteristics of the HRCs observed in some bursts.

In addition, we find that the observed hardness ratio tends to be harder at the beginning of the pulses than what the curvature effect could predict and softer at the late time of the pulses. According to the discussion, we tend to believe that this is an evidence showing the existence of intrinsic hard-to-soft radiation which probably be due to the acceleration-to- 
deceleration mode of shocks. Illustration of the corresponding curves of seven other bursts shows that the characteristics of HRCs observed in the three bursts are common (four of seven cases) in FRED pulse sources and the features predicted by the typical soft burst are seen in two cases.

\section{Acknowledgments}

This work was supported by the National Science Fund (10125313), the National Natural Science Foundation of China (No. 10573005 and No. 10273019), and by a Guangdong Provience fund (Q02114). We also thank the Guangzhou Education Bureau and Guangzhou Science and Technology Bureau for financial support.

[1] J. Goodman, Astrophys. J. 308, L47 (1986)

[2] B. Paczynski, Astrophys. J. 308, L43 (1986)

[3] J. H. Krolik and E. A. Pier, Astrophys. J. 373, 277 (1991)

[4] P. Meszaros and M. J. Rees, Astrophys. J. 502, L105 (1998)

[5] C. J. Hailey, F. A. Harrison and K. Mori, Astrophys. J. 520, L25 (1999)

[6] Y.-P. Qin, Astron. Astrophys. 396, 705 (2002)

[7] Y.-P. Qin, Astron. Astrophys. 407, 393 (2003)

[8] M. Tavani, Astrophys. J. 466, 768 (1996)

[9] N. Lloyd and V. Petrosian, Astrophys. J. 543, 722 (2000)

[10] A. Panaitescu and P. Meszaros, Astrophys. J. 544, L17 (2000)

[11] E. P. Liang, Astrophys. J. 491, L15 (1997)

[12] D. Band et al., Astrophys. J. 413, 281 (1993)

[13] G. J. Fishman et al. Astrophys. J. Suppl. Ser. 92, 229 (1994)

[14] J. P. Norris et al., Astrophys. J. 459, 393 (1996)

[15] D. Kocevski, F. Ryde and E. Liang, Astrophys. J. 596, 389 (2003)

[16] E. E. Fenimore, C. D. Madras and S. Nayakshin, Astrophys. J. 473, 998 (1996)

[17] F. Ryde and V. Petrosian, Astrophys. J. 578, 290 (2002)

[18] Y.-P. Qin et al., Astrophys. J. 617, 439 (2004)

[19] J. P. Norris et al., Astrophys. J. 534, 248 (2000)

[20] E. E. Fenimore et al., Astrophys. J. 448, L101 (1995)

[21] R. J. Nemiroff, Astrophys. J. 544, 805 (2000) 
[22] Y.-P. Qin and R.-J. Lu, Mon. Not. R. Astron. Soc. 362, 1085 (2005)

[23] Y.-P. Qin et al., Astrophys. J. 632, 1008 (2005)

[24] R.-F. Shen, L.-M. Song and Z. Li, Mon. Not. R. Astron. Soc. 362, 59 (2005)

[25] R.-J. Lu et al., Mon. Not. R. Astron. Soc. 367, 275 (2006)

[26] L.-W. Jia and Y.-P. Qin, Mon. Not. R. Astron. Soc. 631, L25 (2005)

[27] Z.-Y. Peng et al., Mon. Not. R. Astron. Soc. 368, 1351 (2006)

[28] E. W. Liang et al., Astrophys. J. 646, 351, (2006)

[29] J. P. Norreis et al., Astrophys. J. 301, 213 (1986)

[30] V. E. Kargatis et al. Astrophys. Space Sci. 231, 177 (1995)

[31] F. Ryde and R. Svensson, Astrophys. J. 529, L13 (2000)

[32] F. Ryde and R. Svensson, Astrophys. J. 566, 210 (2002)

[33] L. Borgonovo and F. Ryde, Astrophys. J. 548, 770 (2001)

[34] P. N. Bhat et al., Astrophys. J. 426, 604 (1994)

[35] For full information of the data of the burst and transient source experiment, see the web site of NASA's CGRO Science Support Center, http://cossc.gsfc.nasa.gov/docs/cgro/batse/.

[36] Concatenated 64-ms burst data in ASCII format of the burst and transient source experiment are available at http://cossc.gsfc.nasa.gov/docs/cgro/batse/batseburst/sixtyfour_ms/index.html.

[37] I. Mitrofanov et al., in Gamma-ray bursts - Observations, Analyses and Theories, edited by C. Ho, R. I. Epstein, and E. E. Fenimore (Cambridge University Press, Cambridge, England, 1992), p. 209.

[38] A. S. Friedman and J. S. Bloom, Astrophys. J. 627, 1 (2005)

[39] R. D. Preece et al., Astrophys. J. Suppl. Ser. 126, 19 (2000)

[40] F Ryde et al., Astron. Astrphys. 411, L331 (2003). 

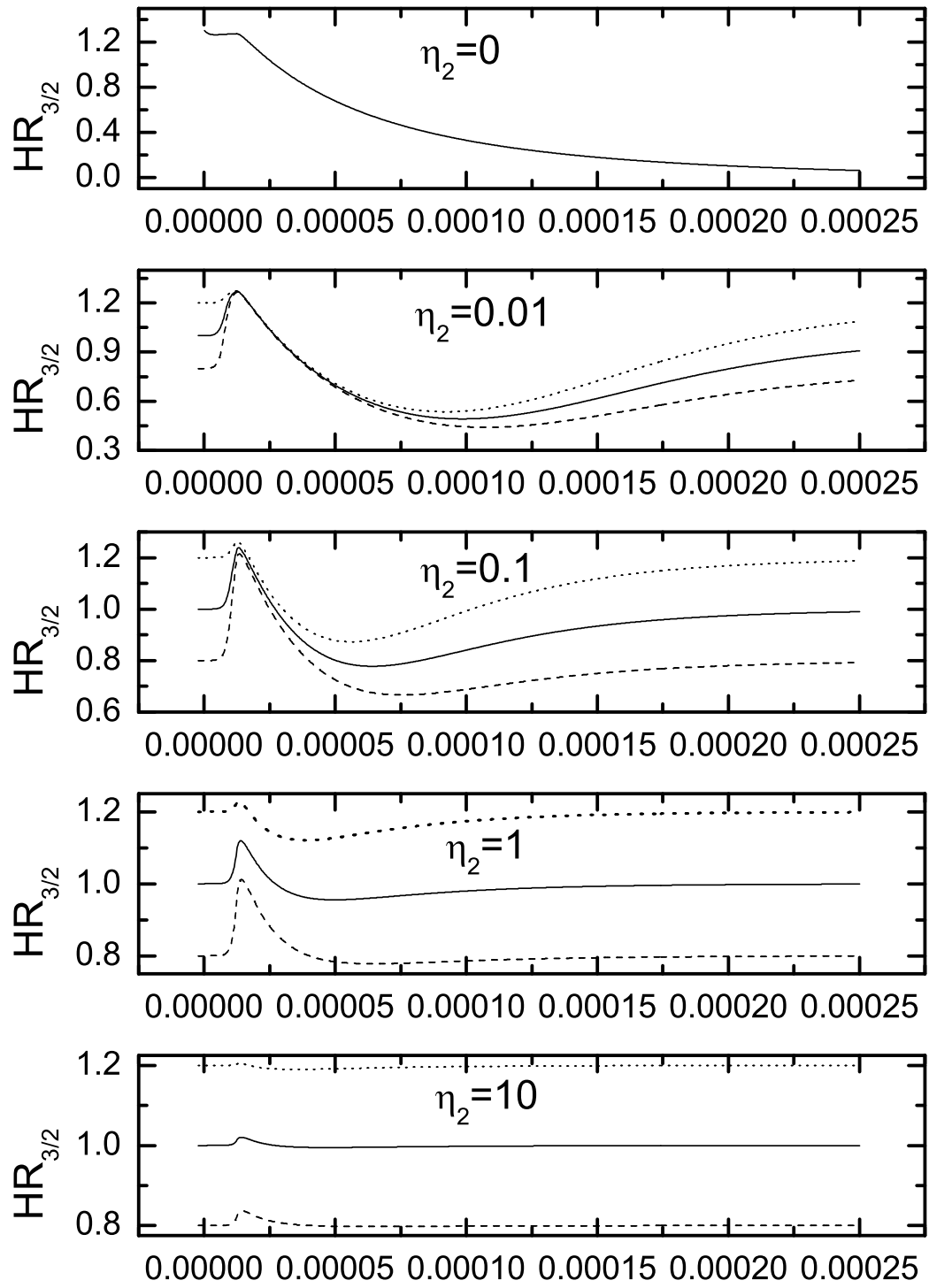

$\tau$

FIG. 4: Plot of the hardness ratio $H R_{3 / 2}$ vs. the relative observation time $\tau$ for various values of $\eta_{2}$ in the case of adopting $\eta_{3}=0.8 \eta_{2}$ (dashed lines), $\eta_{3}=\eta_{2}$ (solid lines), and $\eta_{3}=1.2 \eta_{2}$ (dotted lines), respectively. Other parameters are the same as those adopted in Fig. 1. 

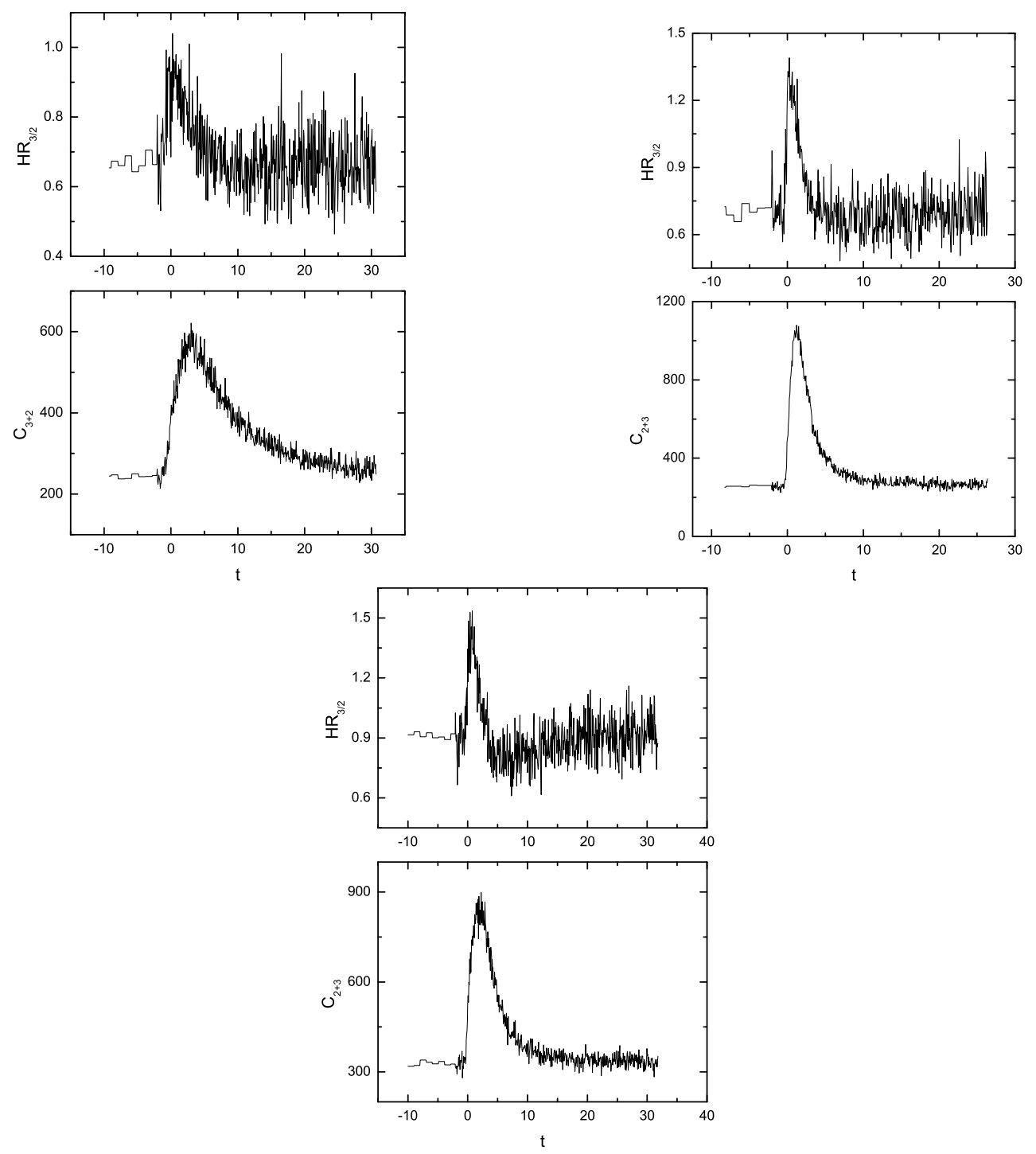

FIG. 5: Developments of the raw hardness ratios (upper panels) and the bi-channel light curves which includes count rates of both the second and third channels (lower panels) of GRB 920216 (top left), GRB 920830 (top right), and GRB 990816 (bottom). 

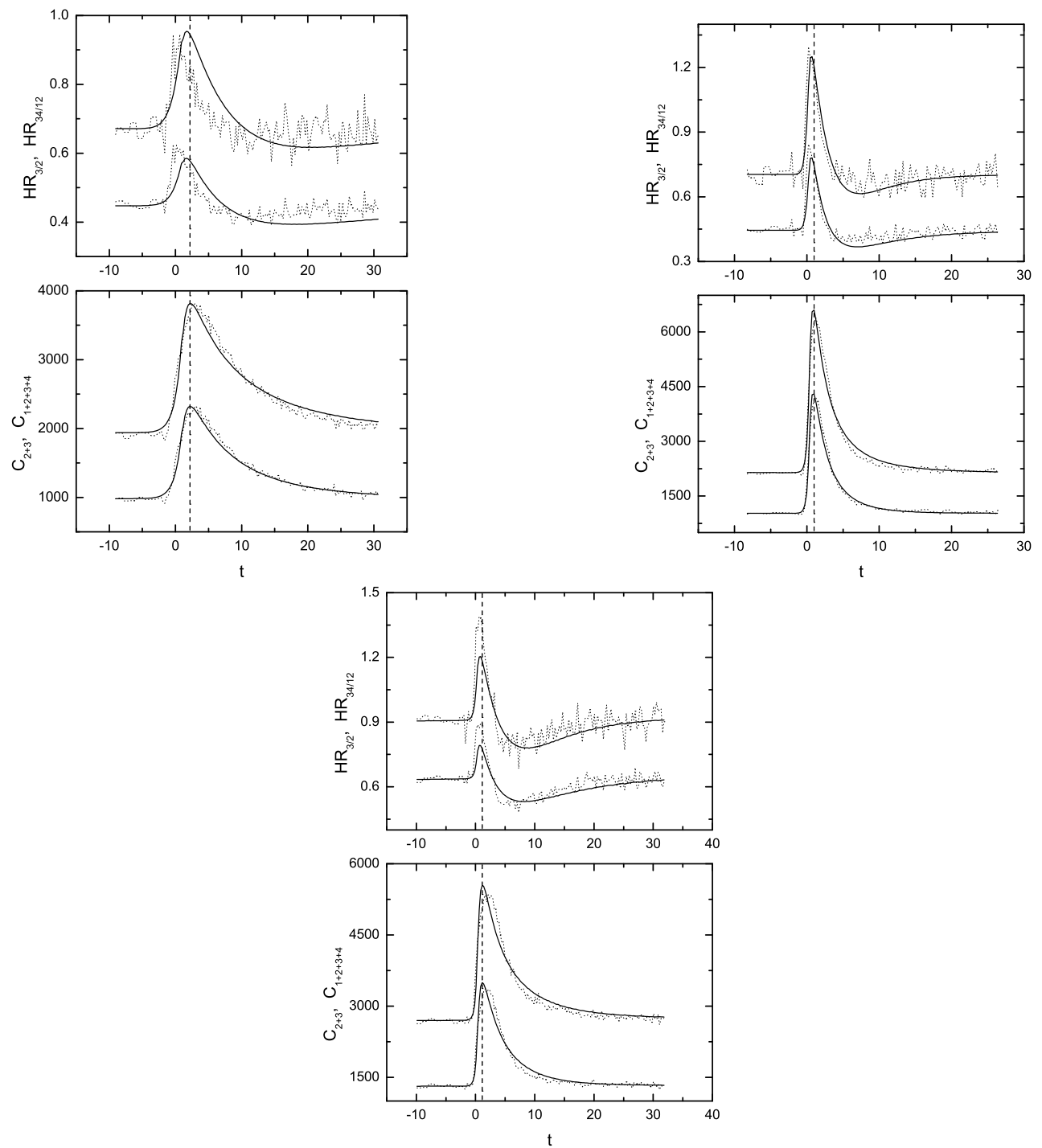

FIG. 6: Fits to the developments of the raw hardness ratios (upper panels) and the multi-channel light curves (lower panels) of GRB 920216 (top left), GRB 920830 (top right) and GRB 990816 (bottom) figure. In the upper panels, solid lines from the bottom to the top represent two expected hardness ratio curves deduced from the four fitting light curves, which are defined by $H R_{34 / 12} \equiv$ $\left(C_{3}+C_{4}\right) /\left(C_{1}+C_{2}\right)$ and $H R_{3 / 2} \equiv C_{3} / C_{2}$, respectively, and dot lines from the bottom to the top stand for the observed data of the corresponding hardness ratio curves respectively. In the lower panels, solid lines from the bottom to the top represent two expected multi-channel light curves deduced from the four fitting light curves, which are defined by $C_{2+3} \equiv C_{2}+C_{3}$ and $C_{1234} \equiv\left(C_{1}+C_{2}+C_{3}+C_{4}\right)$, respectively, and dot lines from the bottom to the top stand for the observed data of the corresponding multi-channel light curves respectively. The dash line in this figure denotes the position of the peak of the expected curve of $C_{2+3}$. 

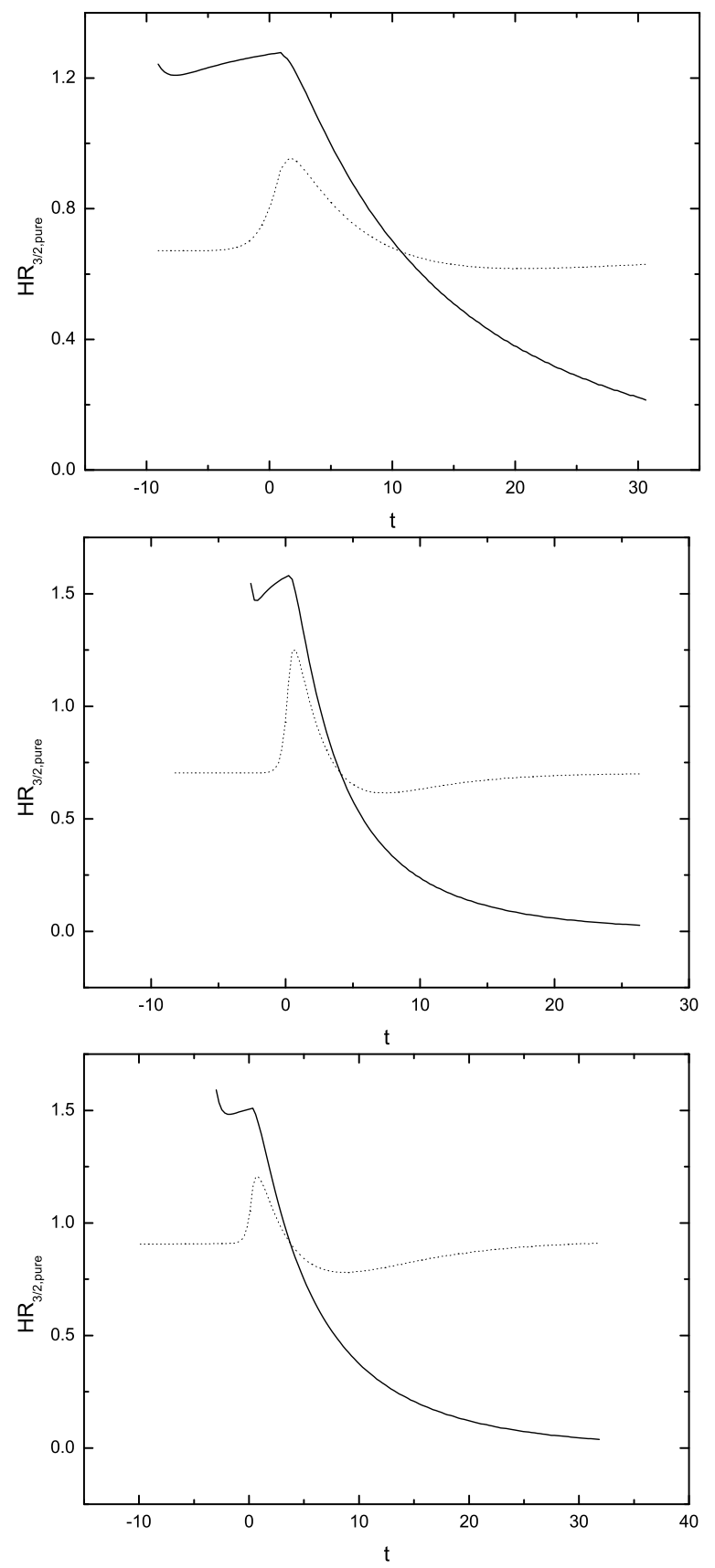

FIG. 7: Developments of the pure hardness ratios (the solid line) and raw hardness ratio curves (the dotted line) for the three sources GRB 920216, GRB 920830 and GRB 990816 (from top to bottom). 

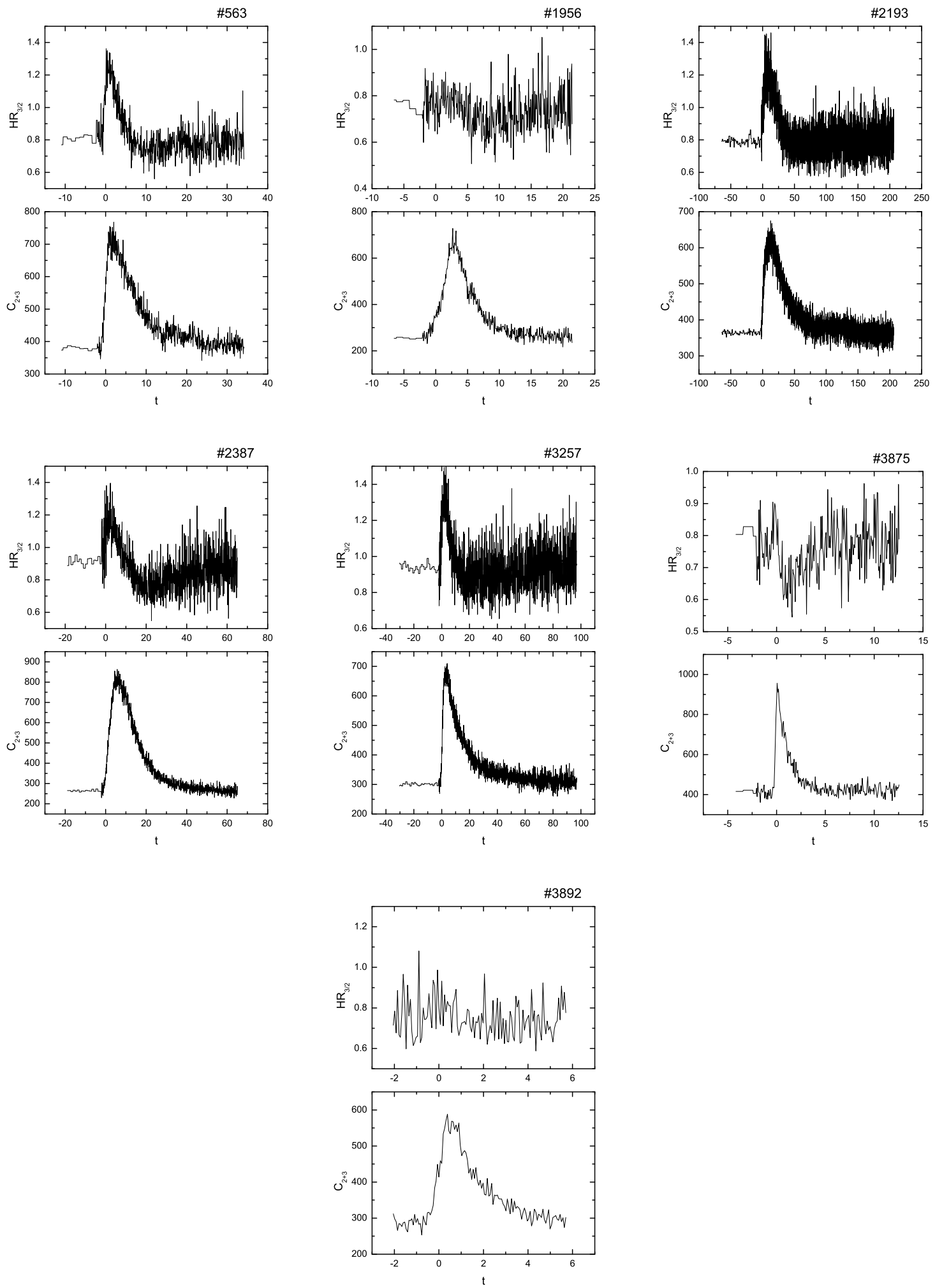

FIG. 8: Developments of the raw hardness ratios and the bi-channel light curves which includes count rates of both the second and third channels of the seven bursts. 